\title{
Research Article Synchronized Hopf Bifurcation Analysis in a Delay-Coupled Semiconductor Lasers System
}

\author{
Gang Zhu' ${ }^{1,2}$ and Junjie Wei ${ }^{1}$ \\ ${ }^{1}$ Department of Mathematics, Harbin Institute of Technology, Heilongjiang, Harbin 150001, China \\ ${ }^{2}$ School of Education Science, Harbin University, Heilongjiang, Harbin 150086, China \\ Correspondence should be addressed to Junjie Wei, weijj@hit.edu.cn
}

Received 30 May 2012; Revised 26 August 2012; Accepted 29 August 2012

Academic Editor: Nazim Idrisoglu Mahmudov

Copyright (c) 2012 G. Zhu and J. Wei. This is an open access article distributed under the Creative Commons Attribution License, which permits unrestricted use, distribution, and reproduction in any medium, provided the original work is properly cited.

The dynamics of a system of two semiconductor lasers, which are delay coupled via a passive relay within the synchronization manifold, are investigated. Depending on the coupling parameters, the system exhibits synchronized Hopf bifurcation and the stability switches as the delay varies. Employing the center manifold theorem and normal form method, an algorithm is derived for determining the Hopf bifurcation properties. Some numerical simulations are carried out to illustrate the analysis results.

\section{Introduction}

Systems of coupled semiconductor lasers (SLs) are receiving increasing interest, because of their practical importance in more and more complex experimental devices, so coupled system are studied by many researchers [1-3]. Moreover, they are important examples of delay-coupled oscillators in general $[4,5]$. The distance between the lasers always results in a time delay in the coupling. In many situations, the time delay has been neglected. However, for semiconductor lasers, this is not always justified due to their large bandwidth and fast time scales of their dynamics. It is well known that delay effects can destabilize a system, furthermore, delay may even result in chaotic dynamics as was shown in [6-8]. On the other hand, time delay in the coupling can also be used to stabilize a chaotic system [9-12]. Synchronization phenomena are common in coupled semiconductor lasers systems. Research shows that even if several individual systems behave chaotically, in the case where the systems are identical, by proper coupling, the systems can be made to evolve toward a situation of isochronal synchronism [13-18]. 
In this paper, we consider the Lang-Kobayashi rate equation [19-22]:

$$
\begin{aligned}
\dot{E}_{j} & =\frac{1}{2}(1+i \alpha) n_{j} E_{j}+k e^{i \varphi} E_{Y}\left(t-\frac{\tau}{2}\right), \\
T \dot{n}_{j} & =p-n_{j}-\left(1+n_{j}\right)\left|E_{j}\right|^{2}, \quad j=1,2,
\end{aligned}
$$

which has already been analyzed by many authors during these years from the physics point of views. Here, $E_{j}$ and $E_{Y}$ are the complex electric field amplitudes of the $j$ th system and the relay, respectively, $n_{j}$ is the excess carrier density, $\alpha$ is the linewidth enhancement factor, $k$ is the coupling strength, $p$ is the pump current, and the time scale parameter $T=\tau_{c} / \tau_{p}$ is the ratio of the carrier and the photon lifetime, $\varphi$ is the feedback phase, and all the parameters in system (1.1) are constants.

In this paper, for the passive relay $E_{Y}$ (realized through a semitransparent mirror, which only receives, reflects, and passes part of the laser from $E_{1}$ and $E_{2}$ ), we consider the algebraic equation:

$$
E_{Y}(t)=\frac{1}{2}\left[E_{1}\left(t-\frac{\tau}{2}\right)+E_{2}\left(t-\frac{\tau}{2}\right)\right]
$$

Noticing the coefficient of the relay $E_{Y}$, we found that since $k, e, i$, and $\varphi$ are all positive constants, so similar to [22], we choose the feedback phase $\varphi=0$ for simplicity, whose results are different from those of the system with $\varphi \neq 0$ only by a constant multiple. Splitting the complex electric field $E_{j}=x_{j}+i y_{j}$ and using the vector $X_{j}=\left(x_{j}, y_{j}, n_{j}\right), j=1,2$, we consider the dynamics within the synchronization manifold (SM), that is, $X_{1}(t)=X_{2}(t)=X(t)$, then we have

$$
E_{Y}\left(t-\frac{\tau}{2}\right)=E(t-\tau)
$$

and (1.1) can be written in the following form:

$$
\begin{aligned}
\dot{x}(t) & =\frac{n(t)}{2}[x(t)-\alpha y(t)]+k x(t-\tau), \\
\dot{y}(t) & =\frac{n(t)}{2}[\alpha x(t)+y(t)]+k y(t-\tau), \\
T \dot{n}(t) & =p-n(t)-[1+n(t)]\left[x^{2}(t)+y^{2}(t)\right] .
\end{aligned}
$$

It is found that, under certain conditions, the equilibrium of system (1.4) is unstable when the delay $\tau$ varies from zero, and as $\tau$ passes through a critical value, the equilibrium becomes asymptotically stable, and after that when $\tau$ passes through another critical value, the equilibrium becomes unstable again, which means that there are stability switches as $\tau$ is increasing. Hence, a Hopf bifurcation occurs at the equilibrium when $\tau$ equals to each critical value, which means system (1.4) has periodic solutions and (1.1) exhibits synchronized periodic oscillation. Since the delay is caused by the distance between the lasers and the 
receiver, we know that the variety of the distance may result in amplitude death (amplitude tending to zero) or periodic oscillation in the complex electric field.

The paper is organized as follows. In Section 2, using the method presented in [23], we study the stability and the existence of Hopf bifurcation of system (1.4) by analyzing the distribution of the roots of the associated characteristic equation, which is a transcendental equation. In Section 3, we use the normal form method and the center manifold theory presented in Hassard et al. [24] to analyze the direction, stability, and period of the bifurcating periodic solution at critical values of $\tau$. In Section 4, some numerical simulations are carried out to illustrate the analytical results.

\section{Stability Analysis}

For (1.4), it is straightforward to see that $E(0,0, p)$ is an equilibrium. Linearizing equation (1.4) around $(0,0, p)$, it follows that

$$
\begin{aligned}
& \dot{x}(t)=\frac{p}{2} x(t)-\frac{\alpha p}{2} y(t)+k x(t-\tau), \\
& \dot{y}(t)=\frac{\alpha p}{2} x(t)+\frac{p}{2} y(t)+k y(t-\tau), \\
& \dot{n}(t)=-\frac{n(t)}{T}
\end{aligned}
$$

whose characteristic equation is given by

$$
\left(\lambda+\frac{1}{T}\right)\left[\left(\lambda-\frac{p}{2}-k e^{-\lambda \tau}\right)^{2}+\frac{\alpha^{2} p^{2}}{4}\right]=0
$$

which is equivalent to the following two equations:

$$
\begin{gathered}
\lambda+\frac{1}{T}=0 \\
\left(\lambda-\frac{p}{2}-k e^{-\lambda \tau}\right)^{2}+\frac{\alpha^{2} p^{2}}{4}=0 .
\end{gathered}
$$

So $\lambda=-(1 / T)$ is always a negative root. Next, we study (2.4).

Obviously, (2.4) is equivalent to

$$
\begin{aligned}
\lambda-\frac{p}{2}-k e^{-\lambda \tau} & =i \frac{\alpha p}{2}, \\
\lambda-\frac{p}{2}-k e^{-\lambda \tau} & =-i \frac{\alpha p}{2} .
\end{aligned}
$$

It is easy to see that the roots of (2.5) are conjugatives of (2.6), so we study (2.5) only. 
When $\tau=0$, we get the root of (2.5) easily

$$
\lambda=\frac{p}{2}+k+i \frac{\alpha p}{2}
$$

so we have

Lemma 2.1. The equilibrium $E(0,0, p)$ is unstable when $\tau=0$.

Let $\lambda=i \omega(\omega \neq 0)$ be a root of (2.5) and substitute $\lambda=i \omega$ into (2.5) yields

$$
\begin{gathered}
\frac{p}{2}+k \cos \omega \tau=0 \\
\omega+k \sin \omega \tau=\frac{\alpha p}{2}
\end{gathered}
$$

which leads to

$$
\left(\omega-\frac{\alpha p}{2}\right)^{2}=k^{2}-\frac{p^{2}}{4}
$$

Then, one gets

$$
\omega_{ \pm}=\frac{1}{2}\left(\alpha p \pm \sqrt{4 k^{2}-p^{2}}\right)
$$

Hence,

$$
\begin{aligned}
& \cos \omega_{+} \tau=\frac{-p}{2 k}, \quad \sin \omega_{+} \tau=\frac{\alpha p-2 \omega_{+}}{2 k}=-\frac{\sqrt{4 k^{2}-p^{2}}}{2 k}, \\
& \cos \omega_{-} \tau=\frac{-p}{2 k}, \quad \sin \omega_{-} \tau=\frac{\alpha p-2 \omega_{-}}{2 k}=\frac{\sqrt{4 k^{2}-p^{2}}}{2 k} .
\end{aligned}
$$

Consequently, for $k>(p / 2)$, one has

$$
\begin{aligned}
& \tau_{j}^{-}=\frac{1}{\omega_{-}}\left(\pi-\arcsin \frac{\sqrt{4 k^{2}-p^{2}}}{2 k}+2 j \pi\right), \\
& \tau_{j}^{+}=\frac{1}{\omega_{+}}\left(\pi+\arcsin \frac{\sqrt{4 k^{2}-p^{2}}}{2 k}+2 j \pi\right), \quad j=0,1,2, \ldots
\end{aligned}
$$


Let

$$
\lambda(\tau)=\gamma(\tau)+i \omega(\tau)
$$

be the root of (2.5) satisfying $\gamma\left(\tau_{j}^{ \pm}\right)=0, \omega\left(\tau_{j}^{ \pm}\right)=\omega_{ \pm}$.

We have the following conclusion.

Lemma 2.2. It holds that

(i) $\gamma^{\prime}\left(\tau_{j}^{+}\right)>0$

(ii) $\gamma^{\prime}\left(\tau_{j}^{-}\right)\left\{\begin{array}{l}<0, \text { when } \frac{p}{2}<k<\frac{\sqrt{1+\alpha^{2}}}{2} p \\ >0, \text { when } k>\frac{\sqrt{1+\alpha^{2}}}{2} p .\end{array}\right.$

Proof. Substituting $\lambda(\tau)$ into (2.5) and taking the derivative with respect to $\tau$, it follows that

$$
\frac{\mathrm{d} \lambda}{\mathrm{d} \tau}+\lambda k e^{-\lambda \tau}+k \tau e^{-\lambda \tau} \frac{\mathrm{d} \lambda}{\mathrm{d} \tau}=0
$$

Therefore, noting that $k e^{-\lambda \tau}=\lambda-(p / 2)-(i / 2) \alpha p$, we have

$$
\frac{\mathrm{d} \lambda}{\mathrm{d} \tau}=\frac{-\lambda(\lambda-(p / 2)-(i / 2) \alpha p)}{1+\tau(\lambda-(p / 2)-(i / 2) \alpha p)}
$$

and by a straight computation, we get

$$
\gamma^{\prime}\left(\tau_{j}^{ \pm}\right)=\frac{\omega_{ \pm}}{\Delta}\left(\omega_{ \pm}-\frac{\alpha p}{2}\right)= \pm \frac{\omega_{ \pm}}{\Delta} \sqrt{k^{2}-\frac{p^{2}}{4}}
$$

where $\Delta=(1+(p \tau / 2))^{2}+\tau^{2}(\omega-(\alpha p / 2))^{2}$. Notice that $\omega_{-}>0$ when $(p / 2)<k<\left(\sqrt{1+\alpha^{2}} / 2\right) p$ and $\omega_{-}<0$ when $k>\left(\sqrt{1+\alpha^{2}} / 2\right) p$, this completes the proof.

As to the order of the sequence $\left\{\tau_{j}^{ \pm}\right\}$, we have

Lemma 2.3. Suppose $(p / 2)<k<(\alpha p / 2 \pi)$. Then, $\tau_{0}^{-}<\tau_{0}^{+}$, and there exists an integer $m \geq 0$ such that

$$
\tau_{0}^{-}<\tau_{0}^{+}<\tau_{1}^{-}<\cdots<\tau_{m}^{-}<\tau_{m}^{+}<\tau_{m+1}^{+}<\tau_{m+1}^{-}
$$


Proof. Condition $(p / 2)<k<(\alpha p / 2 \pi)$ implies that $\tau_{j}^{ \pm}$are well defined. So it is sufficient to verify that $\tau_{0}^{-}<\tau_{0}^{+}$. From

$$
\arcsin x=x+\sum_{n=1}^{\infty} \frac{(2 n-1) ! !}{(2 n+1)(2 n) ! !} x^{2 n+1}=: x+A, \quad x \in[-1,1]
$$

and (2.12), we have

$$
\begin{gathered}
\tau_{0}^{-}=\frac{1}{\omega_{-}}\left(\pi-\frac{\sqrt{4 k^{2}-p^{2}}}{2 k}-A\right), \\
\tau_{0}^{+}=\frac{1}{\omega_{+}}\left(\pi+\frac{\sqrt{4 k^{2}-p^{2}}}{2 k}+A\right),
\end{gathered}
$$

and if $\tau_{0}^{-}<\tau_{0}^{+}$, then

$$
\omega_{+}\left(\pi-\frac{\sqrt{4 k^{2}-p^{2}}}{2 k}-A\right)<\omega_{-}\left(\pi+\frac{\sqrt{4 k^{2}-p^{2}}}{2 k}+A\right)
$$

which is equivalent to

$$
\left(\alpha p+\sqrt{4 k^{2}-p^{2}}\right)\left(\pi-\frac{\sqrt{4 k^{2}-p^{2}}}{2 k}-A\right)<\left(\alpha p-\sqrt{4 k^{2}-p^{2}}\right)\left(\pi+\frac{\sqrt{4 k^{2}-p^{2}}}{2 k}+A\right)
$$

if and only if

$$
\pi \sqrt{4 k^{2}-p^{2}}<\alpha p\left(\frac{\sqrt{4 k^{2}-p^{2}}}{2 k}+A\right)
$$

So, from the condition above, we have

$$
k<\frac{\alpha p}{2 \pi} \Longrightarrow \pi \sqrt{4 k^{2}-p^{2}}<\alpha p \frac{\sqrt{4 k^{2}-p^{2}}}{2 k}<\alpha p\left(\frac{\sqrt{4 k^{2}-p^{2}}}{2 k}+A\right)
$$

This implies that $\tau_{0}^{-}<\tau_{0}^{+}$. 
Journal of Applied Mathematics

$$
\begin{gathered}
\text { From } \tau_{j+1}^{+}-\tau_{j}^{+}=\left(2 \pi / \omega_{+}\right), \tau_{j+1}^{-}-\tau_{j}^{-}=\left(2 \pi / \omega_{-}\right) \text {, and } \omega_{+}>\omega_{-} \text {, we have } \\
\tau_{j+1}^{+}-\tau_{j}^{+}<\tau_{j+1}^{-}-\tau_{j}^{-} .
\end{gathered}
$$

Hence, the conclusion follows.

For convenience, we make the following assumption:

$\left(\mathrm{H}_{1}\right) k \in(0,(p / 2)) \cup\left[\left(\sqrt{1+\alpha^{2}} / 2\right) p, \infty\right)$,

$\left(\mathrm{H}_{2}\right)(p / 2)<k<(\alpha p / 2 \pi)$.

From lemmas $2.1-2.3$ and the fact that $(\alpha p / 2 \pi)<\left(\sqrt{1+\alpha^{2}} / 2\right) p$, then by the Hopf bifurcation theorem for functional differential equations [25], we have the following results on stability and bifurcation to system (1.4).

Theorem 2.4. For system (1.4), the following hold.

(i) If $\left(\mathrm{H}_{1}\right)$ is satisfied, then $E$ is unstable for all $\tau \geq 0$.

(ii) If $\left(\mathrm{H}_{2}\right)$ is satisfied, then system (1.4) undergoes a Hopf bifurcation at $E$ when $\tau=\tau_{j}^{ \pm}$, $j=0,1, \ldots$. Particularly, there exists an integer $m \geq 0$ such that $E$ is unstable when $\tau \in\{0\} \cup\left(\cup_{j=0}^{m}\left(\tau_{j-1}^{+}, \tau_{j}^{-}\right)\right) \cup\left(\tau_{m}^{+}, \infty\right)$ with $\tau_{-1}^{+}=0$, and asymptotically stable when $\tau \in$ $\cup_{j=0}^{m}\left(\tau_{j}^{-}, \tau_{j}^{+}\right)$.

Remark 2.5. From Lemmas 2.1-2.3, we have that all other roots, except $i \omega_{-}\left(\right.$Res. $\left.i \omega_{+}\right)$, of (2.5) with $\tau=\tau_{j}^{-}$(resp., $\left.\tau=\tau_{j}^{+}\right)$have negative real parts for $j=0,1, \ldots, m$ when $\left(\mathrm{H}_{2}\right)$ holds.

\section{The Direction and Stability of Hopf Bifurcation}

In Section 2, we obtained that, under the assumption $\left(\mathrm{H}_{2}\right)$, system (1.4) undergoes a Hopf bifurcation at some critical values of $\tau$. In this section, we study the direction, stability, and the period of the bifurcating periodic solutions. The method we used is based on the normalform method and the center manifold theory presented by Hassard et al. [24].

Transform $E(0,0, p)$ to the origin $O(0,0,0)$ and rescale the time by $t \rightarrow(t / \tau)$ to normalize the delay so that system (1.4) can be written as the form

$$
\begin{aligned}
& \dot{x}(t)=\tau\left[\frac{p}{2}[x(t)-\alpha y(t)]+k x(t-1)+\frac{n(t)}{2}[x(t)-\alpha y(t)]\right], \\
& \dot{y}(t)=\tau\left[\frac{p}{2}[\alpha x(t)+y(t)]+k y(t-1)+\frac{n(t)}{2}[\alpha x(t)+y(t)]\right], \\
& \dot{n}(t)=\frac{\tau}{T}\left[-n(t)-(1+p+n(t))\left(x^{2}(t)+y^{2}(t)\right)\right] .
\end{aligned}
$$

Clearly, the phase space is $\mathcal{C}=\mathcal{C}\left([-1,0], \mathbb{R}^{3}\right)$. For convenience, let $\tau=\tau_{0}+\mu, \mu \in \mathbb{R}$ and $\tau_{0}$ be taken in $\left\{\tau_{j}^{+}\right\} \cup\left\{\tau_{j}^{-}\right\}$. From the analysis in Section 2 , we know that $\mu=0$ is the Hopf bifurcation value for system (3.1), and $i \omega_{0} \tau_{0}$ is the root of the characteristic equation 
associated with the linearization of system (3.1) when $\tau=\tau_{0}$, where either $\omega_{0}=\omega_{+}$or $\omega_{0}=$ $\omega_{-}$. For $\phi=\left(\phi_{1}, \phi_{2}, \phi_{3}\right) \in \mathcal{C}$, let

$$
L_{\mu}(\phi)=\left(\tau_{0}+\mu\right) B \phi(0)+\left(\tau_{0}+\mu\right) C \phi(-1),
$$

where

$$
B=\left(\begin{array}{ccc}
\frac{p}{2} & -\frac{\alpha p}{2} & 0 \\
\frac{\alpha p}{2} & \frac{p}{2} & 0 \\
0 & 0 & -\frac{1}{T}
\end{array}\right), \quad C=\left(\begin{array}{ccc}
k & 0 & 0 \\
0 & k & 0 \\
0 & 0 & 0
\end{array}\right)
$$

By the Rieze representation theorem, there exists a $3 \times 3$ matrix, $\eta(\theta, \mu)(-1 \leq \theta \leq 0)$, whose elements are of bounded variation functions such that

$$
L_{\mu}(\phi)=\int_{-1}^{0} \mathrm{~d} \eta(\theta, \mu) \phi(\theta), \quad \phi \in \mathcal{C}
$$

In fact, we can choose

$$
\eta(\theta, \mu)= \begin{cases}\left(\tau_{0}+\mu\right) B, & \theta=0 \\ 0, & \theta \in(-1,0) \\ -\left(\tau_{0}+\mu\right) C, & \theta=-1\end{cases}
$$

Then, (3.1) is satisfied.

For $\phi \in \mathcal{C}$, define the operator $A(\mu)$ as

$$
A(\mu) \phi(\theta)= \begin{cases}\frac{\mathrm{d} \phi(\theta)}{\mathrm{d} \theta}, & \theta \in[-1,0), \\ \int_{-1}^{0} \mathrm{~d} \eta(t, \mu) \phi(t), & \theta=0,\end{cases}
$$

and $R(\mu) \phi$ as

$$
R(\mu) \phi(\theta)= \begin{cases}0, & \theta \in[-1,0) \\ f(\mu, \phi), & \theta=0\end{cases}
$$


where

$$
f(\mu, \phi)=\left(\tau_{0}+\mu\right)\left(\begin{array}{l}
\frac{\phi_{1}(0) \phi_{3}(0)}{2}-\frac{\alpha \phi_{2}(0) \phi_{3}(0)}{2} \\
\frac{\alpha \phi_{1}(0) \phi_{3}(0)}{2}+\frac{\phi_{2}(0) \phi_{3}(0)}{2} \\
\frac{-1}{T}\left(1+p+\phi_{3}(0)\right)\left(\phi_{1}^{2}(0)+\phi_{2}^{2}(0)\right)
\end{array}\right)
$$

Then, system (3.1) is equivalent to the following operator equation:

$$
\dot{u}_{t}=A(\mu) u_{t}+R(\mu) u_{t}
$$

where $u(t)=(x(t), y(t), n(t))^{T}, u_{t}=u(t+\theta)$, for $\theta \in[-1,0]$.

For $\psi \in \mathcal{C}^{1}\left([0,1], \mathbb{R}^{3}\right)$, define

$$
A^{*} \psi(s)= \begin{cases}-\frac{\mathrm{d} \psi(s)}{\mathrm{d} s}, & s \in(0,1] \\ \int_{-1}^{0} \psi(-\xi) \mathrm{d} \eta(\xi, 0), & s=0 .\end{cases}
$$

For $\phi \in \mathcal{C}[-1,0]$ and $\psi \in \mathcal{C}[0,1]$, define the bilinear form

$$
\langle\psi(s), \phi(\theta)\rangle=\bar{\psi}(0) \phi(0)-\int_{-1}^{0} \int_{0}^{\theta} \bar{\psi}(\xi-\theta) \mathrm{d} \eta(\theta) \phi(\xi) \mathrm{d} \xi,
$$

where $\eta(\theta)=\eta(\theta, 0)$. Then, $A(0)$ and $A^{*}$ are adjoint operators.

Let $q(\theta), q^{*}(s)$ be the eigenvectors of $A(0)$ and $A^{*}$ associated with $i \omega_{0} \tau_{0}$ and $-i \omega_{0} \tau_{0}$, respectively. It is not difficult to verify that

$$
q(\theta)=(1, \beta, 0)^{T} e^{i \omega_{0} \tau_{0} \theta}, \quad q^{*}(s)=\frac{1}{\bar{D}}(1, v, 0) e^{i \omega_{0} \tau_{0} s}
$$

where

$$
\begin{aligned}
\beta & =\frac{-2}{\alpha p}\left(i \omega_{0}-\frac{p}{2}-k e^{-i \omega_{0} \tau_{0}}\right), \\
v & =\frac{-2}{\alpha p}\left(i \omega_{0}+\frac{p}{2}+k e^{i \omega_{0} \tau_{0}}\right), \\
D & =(1+\beta \bar{v})\left(1+k \tau_{0} e^{-i \omega_{0} \tau_{0}}\right),
\end{aligned}
$$

and $\left\langle q^{*}(s), q(\theta)\right\rangle=1,\left\langle q^{*}(s), \bar{q}(\theta)\right\rangle=0$. 
Let $u_{t}$ be the solution of (3.9), and define

$$
z(t)=\left\langle q^{*}, u_{t}\right\rangle, \quad W(t, \theta)=u_{t}(\theta)-2 \operatorname{Re}\{z(t) q(\theta)\}
$$

On the center manifold $\mathfrak{C}_{0}$, we have

$$
W(t, \theta)=W(z(t), \bar{z}(t), \theta)
$$

where

$$
W(z, \bar{z}, \theta)=W_{20} \frac{z^{2}}{2}+W_{11} z \bar{z}+W_{02} \frac{\bar{z}^{2}}{2}+\cdots,
$$

$z$ and $\bar{z}$ are local coordinates for center manifold $\mathfrak{C}_{0}$ in the direction of $q^{*}$ and $\overline{q^{*}}$. Note that $W$ is real if $u_{t}$ is real. We only consider real solutions.

For solution $u_{t}$ in $\mathfrak{C}_{0}$, since $\mu=0$, we have

$$
\begin{aligned}
\dot{z}(t) & =i \omega_{0} \tau_{0} z+\left\langle q^{*}(\theta), f(0, W+2 \operatorname{Re}\{z(t) q(\theta)\})\right\rangle \\
& =i \omega_{0} \tau_{0} z+\overline{q^{*}}(0), f(0, W(z, \bar{z}, 0)+2 \operatorname{Re}\{z(t) q(0)\}) \\
& =i \omega_{0} \tau_{0} z+\overline{q^{*}}(0) f_{0}(z, \bar{z}) .
\end{aligned}
$$

We rewrite this equation as

$$
\dot{z}(t)=i \omega_{0} \tau_{0} z+g(z, \bar{z})
$$

where

$$
g(z, \bar{z})=g_{20} \frac{z^{2}}{2}+g_{11} z \bar{z}+g_{02} \frac{\bar{z}^{2}}{2}+g_{21} \frac{z^{2} \bar{z}}{2} \cdots
$$

By (3.9) and (3.18), we have

$$
\begin{aligned}
\dot{W} & =\dot{u}_{t}-\dot{z} q-\dot{\bar{z}} \bar{q} \\
& = \begin{cases}A W-2 \operatorname{Re}\left\{\overline{q^{*}}(0) f_{0} q(\theta)\right\}, & \theta \in[-1,0), \\
A W-2 \operatorname{Re}\left\{\overline{q^{*}}(0) f_{0} q(0)\right\}+f_{0}, & \theta=0,\end{cases} \\
& =A W+H(z, \bar{z}, \theta),
\end{aligned}
$$

where

$$
H(z, \bar{z}, \theta)=H_{20}(\theta) \frac{z^{2}}{2}+H_{11}(\theta) z \bar{z}+H_{02}(\theta) \frac{\bar{z}^{2}}{2}+\cdots
$$


Journal of Applied Mathematics

Expanding the above series and comparing the coefficients, we obtain

$$
\left(A-2 i \omega_{0} \tau_{0} I\right) W_{20}(\theta)=-H_{20}(\theta), \quad A W_{11}(\theta)=-H_{11}(\theta), \ldots
$$

Noticing that

$$
\begin{gathered}
q(\theta)=(1, \beta, 0)^{T} e^{i \omega_{0} \tau_{0} \theta}, \\
u_{t}(\theta)=z q(\theta)+\bar{z} \bar{q}(\theta)+W(z, \bar{z}, \theta),
\end{gathered}
$$

we have

$$
\begin{gathered}
x(t)=z+\bar{z}+W^{(1)}(z, \bar{z}, 0), \\
y(t)=z \beta+\bar{z} \bar{\beta}+W^{(2)}(z, \bar{z}, 0), \\
x(t-1)=z e^{-i \omega_{0} \tau_{0}}+\bar{z} e^{i \omega_{0} \tau_{0}}+W^{(1)}(z, \bar{z},-1), \\
y(t-1)=z \beta e^{-i \omega_{0} \tau_{0}}+\bar{z} \bar{\beta} e^{i \omega_{0} \tau_{0}}+W^{(2)}(z, \bar{z},-1), \\
n(t)=W^{(3)}(z, \bar{z}, 0),
\end{gathered}
$$

where

$$
\begin{aligned}
& W^{(1)}(z, \bar{z}, \theta)=W_{20}^{(1)}(\theta) \frac{z^{2}}{2}+W_{11}^{(1)}(\theta) z \bar{z}+W_{02}^{(1)}(\theta) \frac{\bar{z}^{2}}{2}+\cdots \\
& W^{(2)}(z, \bar{z}, \theta)=W_{20}^{(2)}(\theta) \frac{z^{2}}{2}+W_{11}^{(2)}(\theta) z \bar{z}+W_{02}^{(2)}(\theta) \frac{\bar{z}^{2}}{2}+\cdots
\end{aligned}
$$

and recalling that

$$
f_{0}=\tau_{0}\left(\begin{array}{l}
\frac{x(t) n(t)}{2}-\frac{\alpha y(t) n(t)}{2} \\
\frac{\alpha x(t) n(t)}{2}+\frac{y(t) n(t)}{2} \\
\frac{-1}{T}(1+p+n(t))\left(x^{2}(t)+y^{2}(t)\right)
\end{array}\right)
$$


we have

$$
\begin{aligned}
& g(z, \bar{z})=\overline{q^{*}}(0) f_{0}=\frac{\tau_{0}}{2 D}\{[x(t) n(t)-\alpha y(t) n(t)]+\bar{\mu}[\alpha x(t) n(t)+y(t) n(t)]\} \\
&=\frac{\tau_{0}}{2 D}\left\{(1+\alpha \bar{v})\left[z+\bar{z}+W_{20}^{(1)}(0) \frac{z^{2}}{2}+W_{11}^{(1)}(0) z \bar{z}+W_{02}^{(1)}(0) \frac{\bar{z}^{2}}{2}+\cdots\right]\right. \\
& \times\left[W_{20}^{(3)}(0) \frac{z^{2}}{2}+W_{11}^{(3)}(0) z \bar{z}+W_{02}^{(3)}(0) \frac{\bar{z}^{2}}{2}+\cdots\right] \\
&+(\bar{v}-\alpha)\left[z \beta+\bar{z} \bar{\beta}+W_{20}^{(2)}(0) \frac{z^{2}}{2}+W_{11}^{(2)}(0) z \bar{z}+W_{02}^{(2)}(0) \frac{\bar{z}^{2}}{2}+\cdots\right] \\
&\left.\times\left[W_{20}^{(3)}(0) \frac{z^{2}}{2}+W_{11}^{(3)}(0) z \bar{z}+W_{02}^{(3)}(0) \frac{\bar{z}^{2}}{2}+\cdots\right]\right\} .
\end{aligned}
$$

We can obtain the coefficients which will be used in determining the important quantities:

$$
\begin{aligned}
& g_{20}=g_{11}=g_{02}=0 \\
& g_{21}=\frac{\tau_{0}}{D}\left[\frac{W_{20}^{(3)}(0)}{2}(1-\alpha \bar{\beta}+\alpha \bar{\nu}+\bar{\beta} \bar{\nu})+W_{11}^{(3)}(0)(1-\alpha \beta+\alpha \overline{\mathcal{v}}+\beta \overline{\mathcal{v}})\right] .
\end{aligned}
$$

We still need to compute $W_{20}(\theta)$ and $W_{11}(\theta)$ for $\theta \in[-1,0)$. We have

$$
H(z, \bar{z}, \theta)=-\bar{q}^{*}(0) f_{0} q(\theta)-q^{*}(0) \overline{f_{0}} \bar{q}(\theta)=-g(z, \bar{z}) q(\theta)-\bar{g}(z, \bar{z}) \bar{q}(\theta)
$$

Comparing the coefficients about $H(z, \bar{z}, \theta)$ gives that

$$
H_{20}(\theta)=-g_{20} q(\theta)-\bar{g}_{02} \bar{q}(\theta)=0, \quad H_{11}=-g_{11} q(\theta)-\bar{g}_{11} \bar{q}(\theta)=0 .
$$

Then, from (3.22), we get

$$
\dot{W}_{20}(\theta)=2 i \omega_{0} \tau_{0} W_{20}(\theta), \quad \dot{W}_{11}(\theta)=0,
$$

which implies that

$$
W_{20}(\theta)=E e^{2 i \omega_{0} \tau_{0} \theta}, \quad W_{11}(\theta)=F,
$$


where $E, F$ are both three-dimensional vectors and can be determined by setting $\theta=0$ in $H(z, \bar{z}, \theta)$. In fact, from

$$
\begin{aligned}
H(z, \bar{z}, 0)= & -2 \operatorname{Re}\left\{\bar{q}^{*}(0) f_{0} q(0)+f_{0}\right\} \\
= & -g(z, \bar{z}) q(0)-\bar{g}(z, \bar{z}) \bar{q}(0)+\frac{\tau_{0}}{2}\left(\begin{array}{c}
x(t) n(t)-\alpha y(t) n(t) \\
\alpha x(t) n(t)+y(t) n(t) \\
\frac{-2}{T}(1+p+n(t))\left(x^{2}(t)+y^{2}(t)\right)
\end{array}\right) \\
= & -g(z, \bar{z}) q(0)-\bar{g}(z, \bar{z}) \bar{q}(0) \\
& +\frac{\tau_{0}}{2}\left(\begin{array}{l}
\left(W^{(1)}+z+\bar{z}\right) W^{(3)}-\alpha\left(W^{(2)}+z \beta+\bar{z} \bar{\beta}\right) W^{(3)} \\
\frac{-2}{T}\left(1+p+W^{(1)}\right)\left[\left(W^{(1)}+z+\bar{z}\right)^{2}+\left(W^{(2)}+z \beta+\bar{z} \bar{\beta}\right)^{2}\right]
\end{array}\right)
\end{aligned}
$$

we have

$$
\begin{aligned}
H_{20}(0) & =-g_{20} q(0)-\bar{g}_{02} \bar{q}(0)+\tau_{0}\left(0,0,-\frac{2}{T}(1+p)\left(1+\beta^{2}\right)\right)^{T} \\
& =\tau_{0}\left(0,0,-\frac{2}{T}(1+p)\left(1+\beta^{2}\right)\right)^{T} \\
H_{11}(0) & =-g_{11} q(0)-\bar{g}_{11} \bar{q}(0)+\tau_{0}\left(0,0,-\frac{2}{T}(1+p)\left(1+|\beta|^{2}\right)\right)^{T} \\
& =\tau_{0}\left(0,0,-\frac{2}{T}(1+p)\left(1+|\beta|^{2}\right)\right)^{T} .
\end{aligned}
$$

It follows from (3.22) and the definition of $A$ that

$$
\begin{aligned}
& \tau_{0} B W_{20}(0)+\tau_{0} C W_{20}(-1)=2 i \omega_{0} \tau_{0} W_{20}(0)-H_{20}(0), \\
& \tau_{0} B W_{11}(0)+\tau_{0} C W_{11}(-1)=-H_{11}(0) .
\end{aligned}
$$

Combining the conditions above, we have

$$
\begin{aligned}
\left(B+e^{-2 i \omega_{0} \tau_{0}} C-2 i \omega_{0} I\right) E & =\left(0,0, \frac{2}{T}(1+p)\left(1+\beta^{2}\right)\right)^{T} \\
(B+C) F & =\left(0,0, \frac{2}{T}(1+p)\left(1+|\beta|^{2}\right)\right)^{T},
\end{aligned}
$$


which implies

$$
\begin{aligned}
& E=\left(0,0, \frac{-2}{1+2 i T \omega_{0}}(1+p)\left(1+\beta^{2}\right)\right)^{T} \\
& F=\left(0,0,-2(1+p)\left(1+|\beta|^{2}\right)\right) .
\end{aligned}
$$

Consequently, the above $g_{21}$ can be expressed by the parameters and delay in system (3.1). Thus, we can compute the following quantities:

$$
\begin{aligned}
c_{1}(0)= & \frac{i}{2 \omega_{0} \tau_{0}}\left(g_{20} g_{11}-2\left|g_{11}\right|^{2}-\frac{1}{3}\left|g_{02}\right|^{2}\right)+\frac{g_{21}}{2} \\
= & \frac{-\tau_{0}(1+p)}{2 D}\left[\frac{1}{1+2 i \omega_{0} T}\left(1+\beta^{2}\right)(1-\alpha \bar{\beta}+\alpha \bar{v}+\bar{\beta} \bar{v})\right. \\
& \left.+2\left(1+|\beta|^{2}\right)(1-\alpha \beta+\alpha \bar{v}+\beta \bar{v})\right], \\
\mu_{2}= & -\frac{\operatorname{Re} c_{1}(0)}{\operatorname{Re} \lambda^{\prime}\left(\tau_{0}\right)}, \\
\beta_{2}= & 2 \operatorname{Re} c_{1}(0), \\
T_{2}= & -\frac{\operatorname{Im} c_{1}(0)+\mu_{2} \operatorname{Im} \lambda^{\prime}\left(\tau_{0}\right)}{\omega_{0} \tau_{0}},
\end{aligned}
$$

which determine the properties of bifurcating periodic solutions at the critical value $\tau_{0}$. The direction and stability of Hopf bifurcation in the center manifold can be determined by $\mu_{2}$ and $\beta_{2}$, respectively. In fact, if $\mu_{2}>0\left(\mu_{2}<0\right)$, then the bifurcating periodic solutions are forward (backward); the bifurcating periodic solutions on the center manifold are stable (unstable) if $\beta_{2}<0\left(\beta_{2}>0\right) ; T_{2}$ determines the period of the bifurcating periodic solutions: the period increases (decreases) if $T_{2}>0\left(T_{2}<0\right)$.

From the discussion in Section 2, we have known that

(i) $\operatorname{Re} \lambda^{\prime}\left(\tau_{j}^{+}\right)>0$,

(ii) $\operatorname{Re} \lambda^{\prime}\left(\tau_{j}^{-}\right)\left\{\begin{array}{l}<0, \quad \text { when } \frac{p}{2}<k<\frac{\sqrt{1+\alpha^{2}}}{2} p, \\ >0, \quad \text { when } k>\frac{\sqrt{1+\alpha^{2}}}{2} p .\end{array}\right.$

From Remark 2.5, we have the following results. 
Theorem 3.1. For system (1.4), suppose that condition $\left(\mathrm{H}_{2}\right)$ is satisfied. Then, when $0 \leq j \leq m$, one has

(i) the Hopf bifurcation at $E(0,0, p)$ when $\tau=\tau_{j}^{-}$is backward (forward) and the bifurcation periodic solutions are stable (unstable) if $\operatorname{Re}\left(c_{1}(0)\right)<0(>0)$;

(ii) the Hopf bifurcation at $E(0,0, p)$ when $\tau=\tau_{j}^{+}$is forward (backward) and the bifurcation periodic solutions are stable (unstable) if $\operatorname{Re}\left(c_{1}(0)\right)<0(>0)$.

Here $\tau_{j}^{ \pm}$and $m$ are given in (ii) of Theorem 2.4 .

\section{Numerical Simulations}

In this section, we will carry out numerical simulation on system (1.4). Set

(A) $\alpha=9.42, k=1, p=1, T=20$.

Clearly, $\left(\mathrm{H}_{2}\right)$ is satisfied. We have $\omega_{-} \doteq 3.845, \omega_{+} \doteq 5.575$ and

$$
\tau_{0}^{-} \doteq 0.5448, \quad \tau_{0}^{+} \doteq 0.7507, \quad \tau_{1}^{+} \doteq 1.8771, \quad \tau_{1}^{-} \doteq 2.1782 \cdots
$$

Thus, the equilibrium $(0,0,1)$ is stable when $\tau \in\left(\tau_{0}^{-}, \tau_{0}^{+}\right)$, and unstable when $\tau \in\left[0, \tau_{0}^{-}\right) \cup$ $\left(\tau_{0}^{+}, \infty\right)$. Furthermore, we get

$$
\operatorname{Re} \lambda^{\prime}\left(\tau_{0}^{-}\right) \doteq-1.8411, \quad \operatorname{Re} \lambda^{\prime}\left(\tau_{0}^{+}\right) \doteq 2.3133
$$

By the algorithm derived in Section 3, we can obtain

$$
\operatorname{Re} c_{1}(0) \doteq-0.7414, \quad \mu_{2} \doteq-0.1027, \quad \beta_{2} \doteq-1.4828
$$

at $\tau=\tau_{0}^{-}$, and

$$
\operatorname{Re} c_{1}(0) \doteq-4.7053, \quad \mu_{2} \doteq 2.0340, \quad \beta_{2} \doteq-9.4106
$$

at $\tau=\tau_{0}^{+}$, respectively. These imply that the direction of Hopf bifurcations is backward when $\tau=\tau_{0}^{-}$, and forward when $\tau=\tau_{0}^{+}$, respectively, and the bifurcating periodic solutions are orbitally asymptotically stable. These are shown in Figures 1, 2, and 3.

\section{Conclusion}

Flunkert et al. [22] explored an experimental system of two semiconductor lasers coupled via a passive relay within the synchronization manifold. They calculated the maximum transversal Lyapunov exponential and got blow-out bifurcations when the coupling strength $k$ passed through critical values.

In this paper, we also study the coupled system realized by a passive relay within the synchronization manifold. By analyzing the distribution of eigenvalues, we study the 


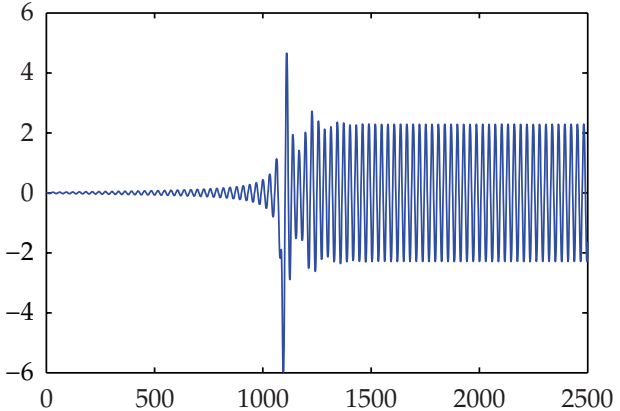

(a)

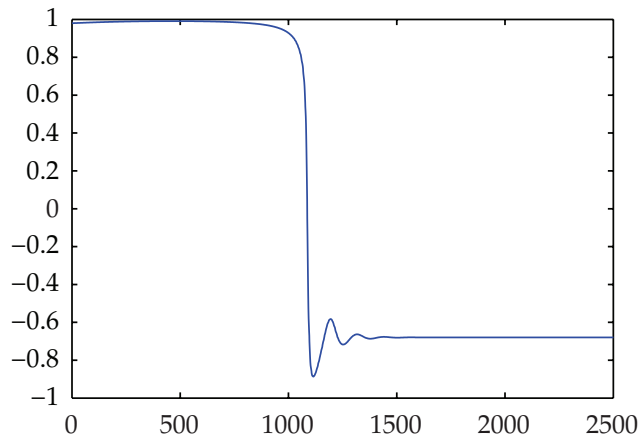

(c)

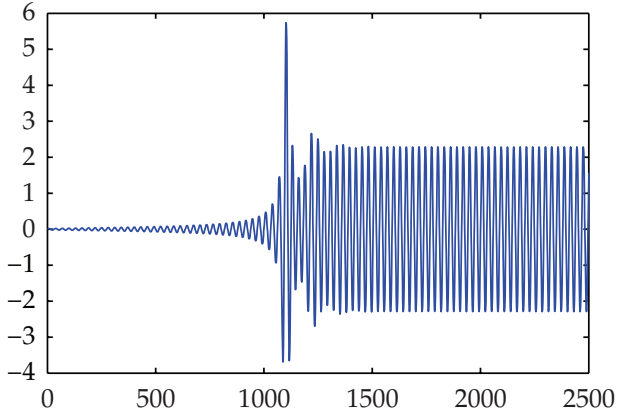

(b)

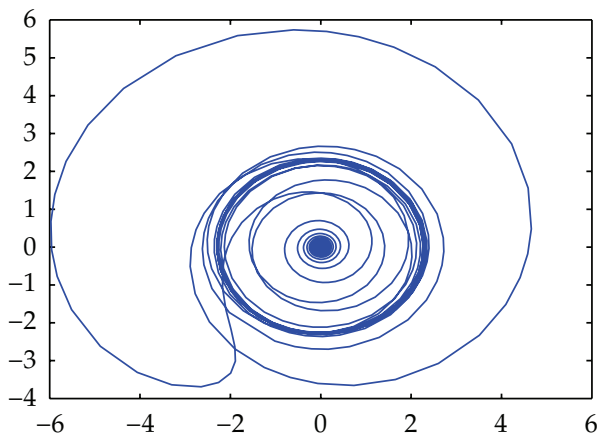

(d)

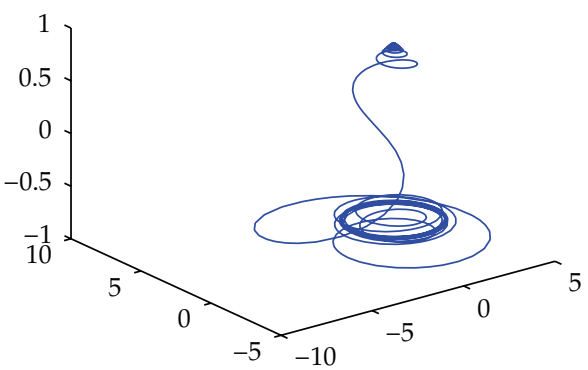

(e)

Figure 1: For system (1.4) with the data (A), the Hopf bifurcation is backward at the first critical value $\tau_{0}^{-} \doteq 0.5448$, and the bifurcating periodic solutions are asymptotically stable, where $\tau=0.54<0.5448$ and the initial value is taken as $(0.01,0.01,0.98)$.

stability of the equilibrium and the existence of periodic solutions. We find that as the coupling strength increases, under the condition $\left(\mathrm{H}_{2}\right)$, the stability switch for $\tau$ occurs, which means that there exists a sequence values of $\tau_{j}^{ \pm}$and an integer $m$ satisfying

$$
0<\tau_{0}^{-}<\tau_{0}^{+}<\tau_{1}^{-}<\cdots<\tau_{m}^{-}<\tau_{m}^{+}<\tau_{m+1}^{+}
$$




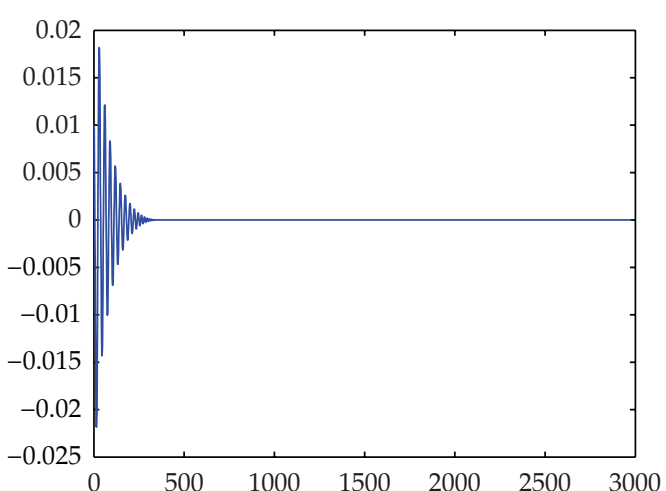

(a)

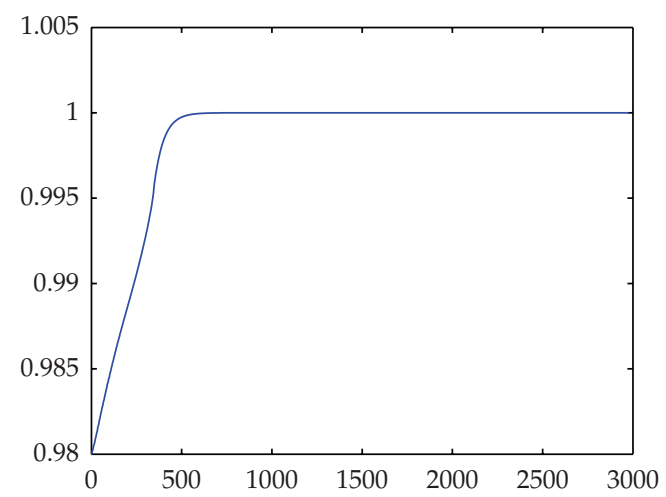

(c)

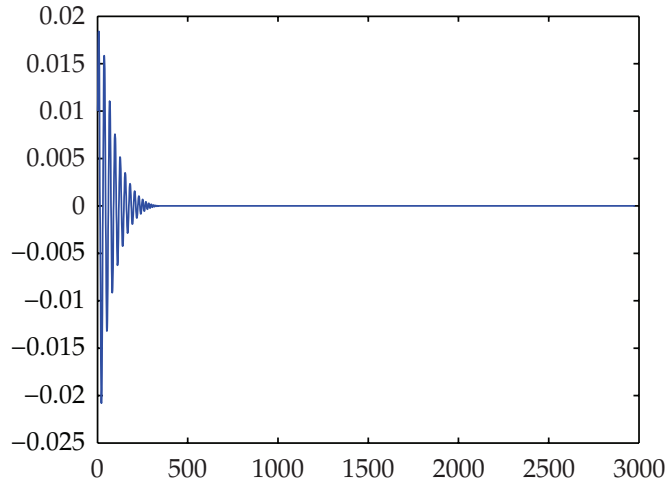

(b)

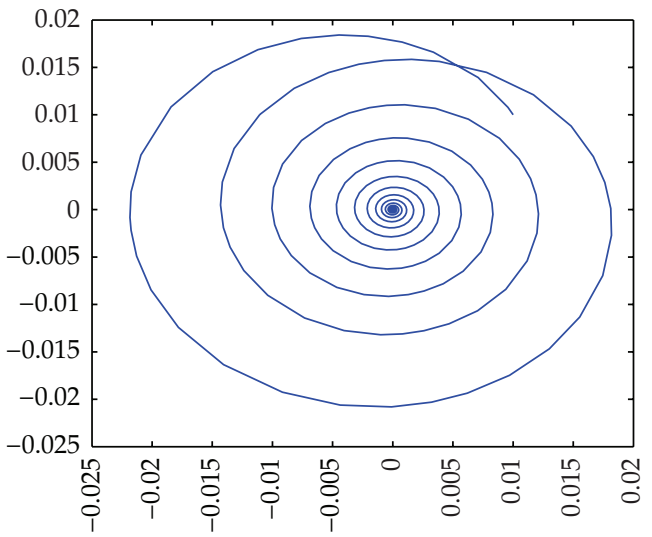

(d)

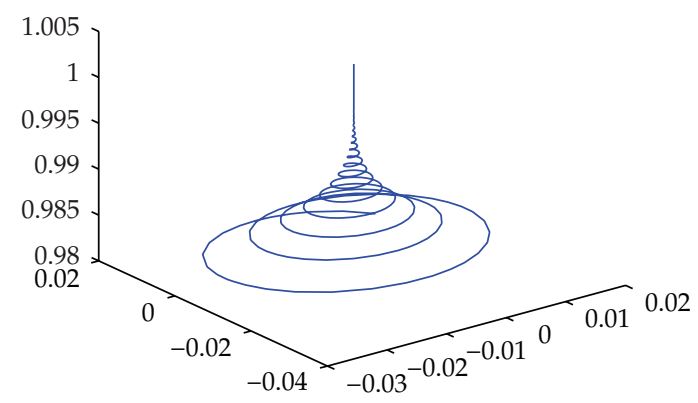

(e)

Figure 2: For system (1.4) with the data (A), the equilibrium $E(0,0,1)$ is asymptotically stable when $\tau \in$ $\left(\tau_{0}^{-}, \tau_{0}^{+}\right)$, where $\tau=0.6$ and the initial value is taken as $(0.01,0.01,0.98)$.

such that the equilibrium $E$ is asymptotically stable when $\tau \in \cup_{j=0}^{m}\left(\tau_{j}^{-}, \tau_{j}^{+}\right)$, and unstable when $\tau \in\{0\} \cup\left(\cup_{j=0}^{m}\left[\tau_{j-1}^{+}, \tau_{j}^{-}\right)\right) \cup\left(\tau_{m}^{+}, \infty\right)$, and the system undergoes a Hopf bifurcation at $\tau=\tau_{j}^{ \pm}$, where $j=1,2, \ldots$.

As a result, the modulation of the coupling strength $k$ and the delay $\tau$ (which is caused by the distance between the lasers and the relay) would be an efficient method to control the 


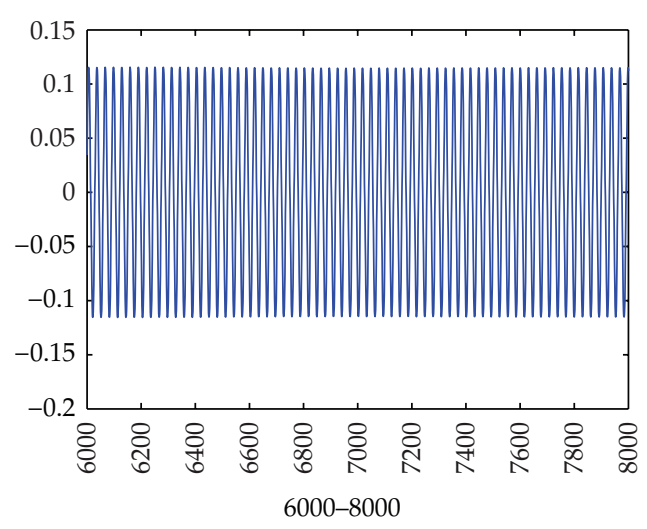

(a)

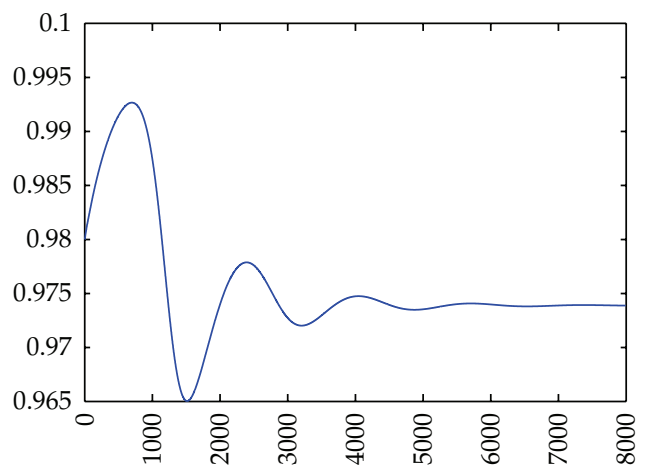

(c)

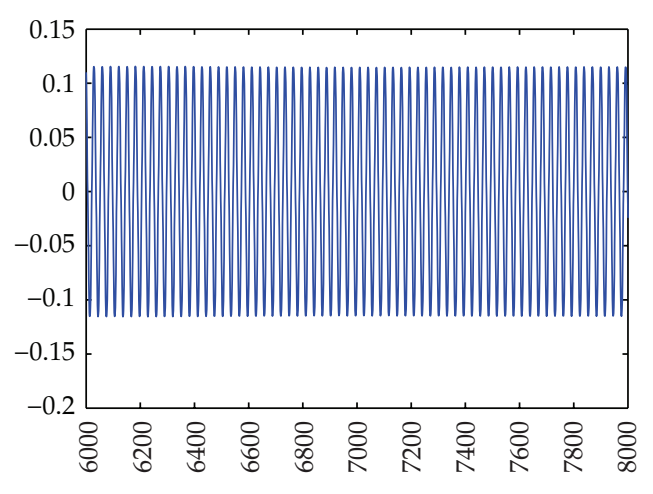

(b)

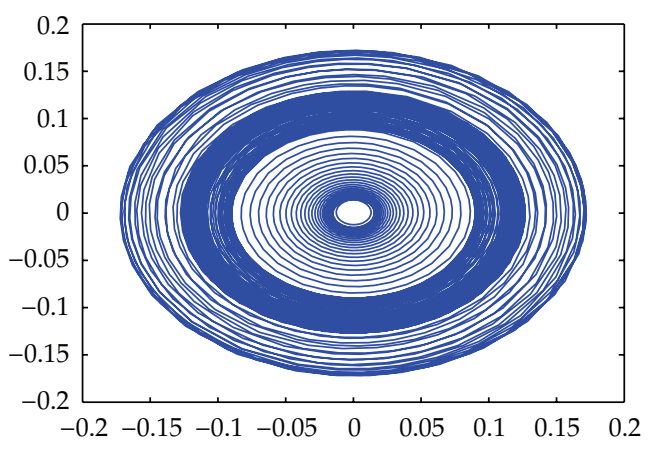

(d)

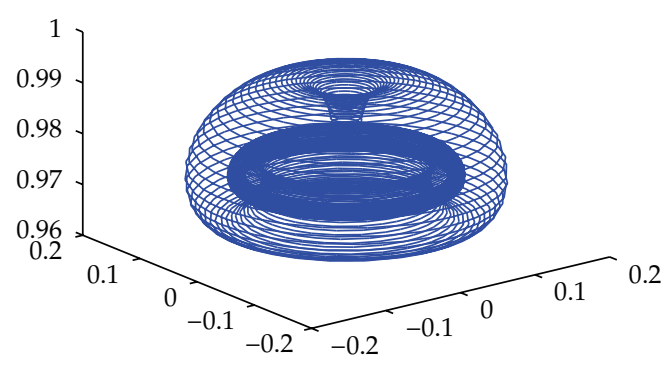

(e)

Figure 3: For system (1.4) with the data (A), the Hopf bifurcation is forward at $\tau_{0}^{+} \doteq 0.7507$, and the bifurcating periodic solutions are asymptotically stable, where $\tau=0.77>\tau_{0}^{+}$and the initial value is taken as $(0.01,0.01,0.98)$.

system in the complex electric field; the amplitude either vanishes or presents a periodic oscillation.

As per the coupled system which is realized by an active relay and the systems without synchronization, we will study in the future. 


\section{Acknowledgments}

The authors are grateful to the anonymous referees for their helpful comments and valuable suggestions. This research is supported by the National Natural Science Foundation of China (no. 11031002).

\section{References}

[1] M. Möhrle, B. Sartorius, C. Bornholdt et al., “Detuned grating multisection-RW-DFB lasers for highspeed optical signal processing," IEEE Journal on Selected Topics in Quantum Electronics, vol. 7, no. 2, pp. 217-223, 2001.

[2] M. T. Hill, H. De Waardt, G. D. Khoe, and H. J. S. Dorren, "All-optical flip-flop based on coupled laser diodes," IEEE Journal of Quantum Electronics, vol. 37, no. 3, pp. 405-413, 2001.

[3] E. F. Manffra, I. L. Caldas, R. L. Viana, and H. J. Kalinowski, “Type-I intermittency and crisis-induced intermittency in a semiconductor laser under injection current modulation," Nonlinear Dynamics, vol. 27, no. 2, pp. 185-195, 2002.

[4] H. Erzgräber, B. Krauskopf, and D. Lenstra, "Compound laser modes of mutually delay-coupled lasers," SIAM Journal on Applied Dynamical Systems, vol. 5, no. 1, pp. 30-65, 2006.

[5] H. Erzgräber, D. Lenstra, B. Krauskopf et al., "Mutually delay-coupled semiconductor lasers: mode bifurcation scenarios," Optics Communications, vol. 255, no. 4-6, pp. 286-296, 2005.

[6] G. Stépán, Retarded Dynamical Systems: Stability and Characteristic Functions, vol. 210, Longman Scientific \& Technical, London, UK, 1989.

[7] T. Heil, I. Fischer, W. Elsäßer, and A. Gavrielides, "Dynamics of semiconductor lasers subject to delayed optical feedback: the short cavity regime," Physical Review Letters, vol. 87, no. 24, Article ID 243901, 2001.

[8] T. Heil, I. Fischer, W. Elsäßer, J. Mulet, and C. R. Mirasso, “Chaos synchronization and spontaneous symmetry-breaking in symmetrically delay-coupled semiconductor lasers," Physical Review Letters, vol. 86, no. 5, pp. 795-798, 2001.

[9] J. Wei and C. Yu, "Stability and bifurcation analysis in the cross-coupled laser model with delay," Nonlinear Dynamics, vol. 66, no. 1, pp. 29-38, 2011.

[10] D. V. Ramana Reddy, A. Sen, and G. L. Johnston, "Time delay induced death in coupled limit cycle oscillators," Physical Review Letters, vol. 80, no. 23, pp. 5109-5112, 1998.

[11] I. Fischer, Y. Liu, and P. Davis, "Synchronization of chaotic semiconductor laser dynamics on subnanosecond time scales and its potential for chaos communication," Physical Review A, vol. 62, no. 1, Article ID 011801, 2000.

[12] B. Ravoori, A. B. Cohen, A. V. Setty et al., "Adaptive synchronization of coupled chaotic oscillators," Physical Review E, vol. 80, no. 5, Article ID 056205, 2009.

[13] A. Arenas, A. Díaz-Guilera, J. Kurths, Y. Moreno, and C. Zhou, "Synchronization in complex networks," Physics Reports, vol. 469, no. 3, pp. 93-153, 2008.

[14] M. Nixon, M. Friedman, E. Ronen, A. A. Friesem, N. Davidson, and I. Kanter, "Synchronized cluster formation in coupled laser networks," Physical Review Letters, vol. 106, no. 22, Article ID 223901, 2011.

[15] X. Liu, Q. Gao, and L. Niu, "A revisit to synchronization of Lurie systems with time-delay feedback control," Nonlinear Dynamics, vol. 59, no. 1-2, pp. 297-307, 2010.

[16] C. F. Feng, "Projective synchronization between two different time-delayed chaotic systems using active control approach," Nonlinear Dynamics, vol. 62, pp. 453-459, 2010.

[17] H. Huang, G. Feng, and J. Cao, “Exponential synchronization of chaotic Lur'e systems with delayed feedback control," Nonlinear Dynamics, vol. 57, no. 3, pp. 441-453, 2009.

[18] J. Lu and J. Cao, "Adaptive synchronization of uncertain dynamical networks with delayed coupling," Nonlinear Dynamics, vol. 53, no. 1-2, pp. 107-115, 2008.

[19] R. Lang and K. Kobayashi, "External optical feedback effects on semiconductor injection laser properties," IEEE Journal of Quantum Electronics, vol. 16, no. 3, pp. 347-355, 1980.

[20] P. M. Alsing, V. Kovanis, A. Gavrielides, and T. Erneux, "Lang and Kobayashi phase equation," Physical Review A, vol. 53, no. 6, pp. 4429-4434, 1996.

[21] J. D. Farmer, "Chaotic attractors of an infinite-dimensional dynamical system," Physica D, vol. 4, no. 3, pp. 366-393, 1982. 
[22] V. Flunkert, O. D’Huys, J. Danckaert, I. Fischer, and E. Schöll, "Bubbling in delay-coupled lasers," Physical Review E, vol. 79, no. 6, Article ID 065201, 2009.

[23] S. Ruan and J. Wei, “On the zeros of transcendental functions with applications to stability of delay differential equations with two delays," Dynamics of Continuous, Discrete $\mathcal{E}$ Impulsive Systems Series A, vol. 10, no. 6, pp. 863-874, 2003.

[24] B. D. Hassard, N. D. Kazarinoff, and Y. H. Wan, Theory and Applications of Hopf Bifurcation, vol. 41, Cambridge University Press, Cambridge, UK, 1981.

[25] J. Hale, Theory of Functional Differential Equations, Springer, New York, NY, USA, 1977. 


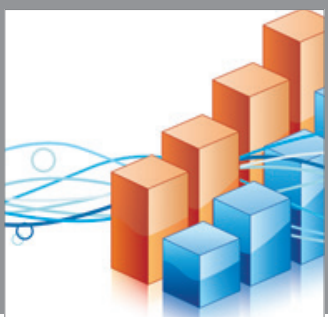

Advances in

Operations Research

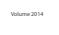

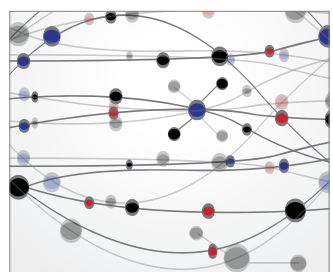

\section{The Scientific} World Journal
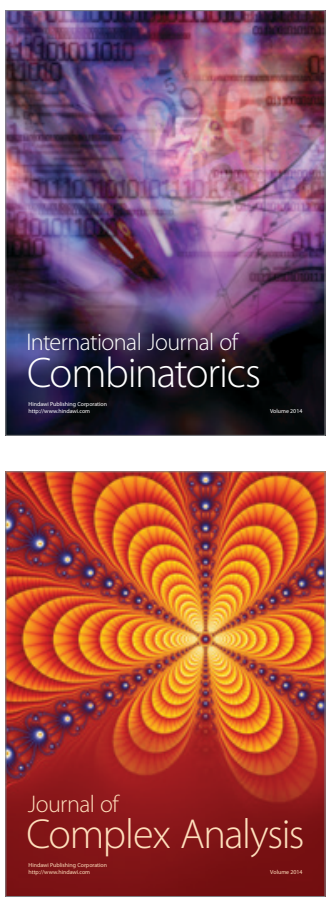

International Journal of

Mathematics and

Mathematical

Sciences
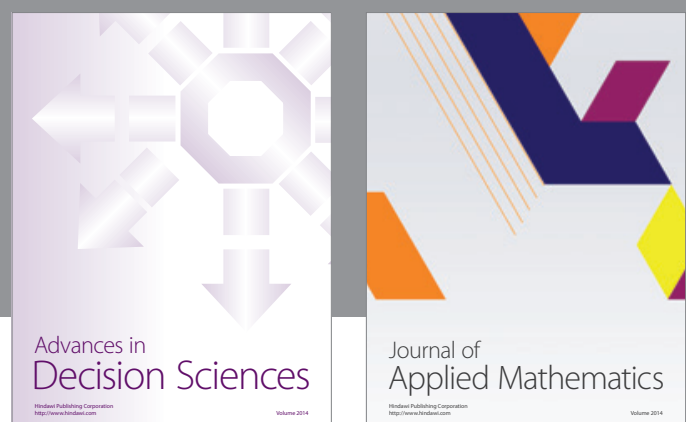

Journal of

Applied Mathematics
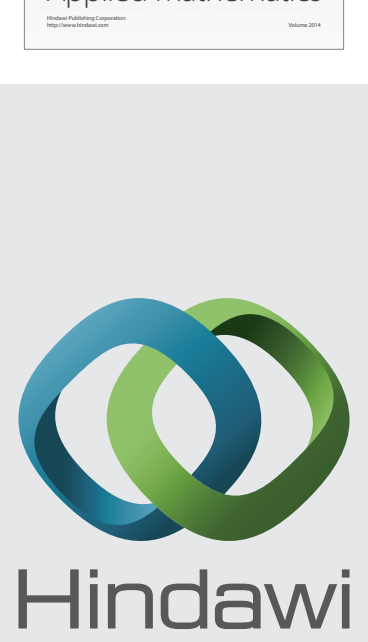

Submit your manuscripts at http://www.hindawi.com
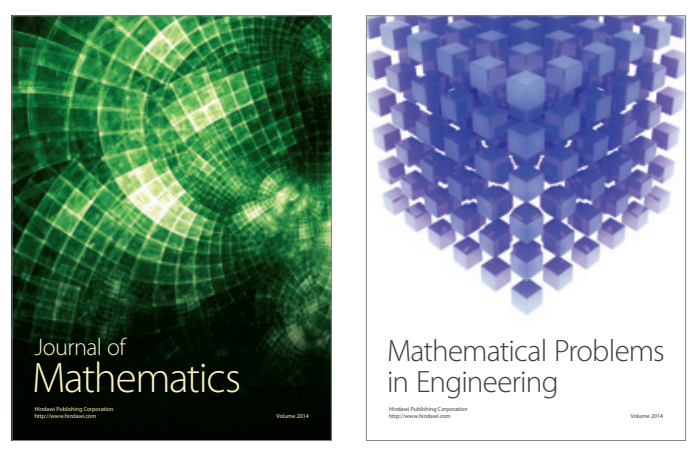

Mathematical Problems in Engineering
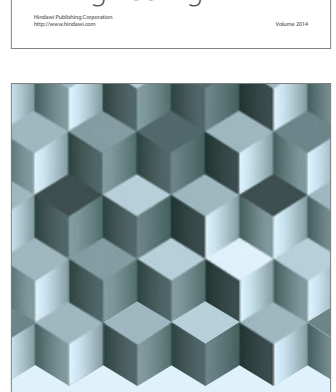

Journal of

Function Spaces
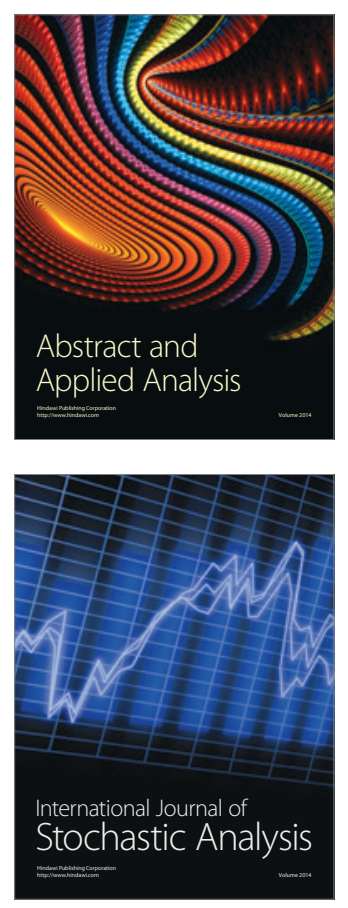

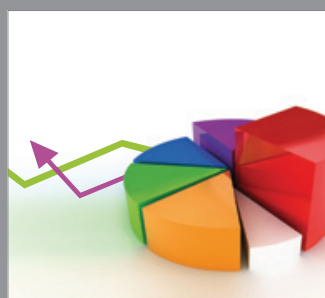

ournal of

Probability and Statistics

Promensencen
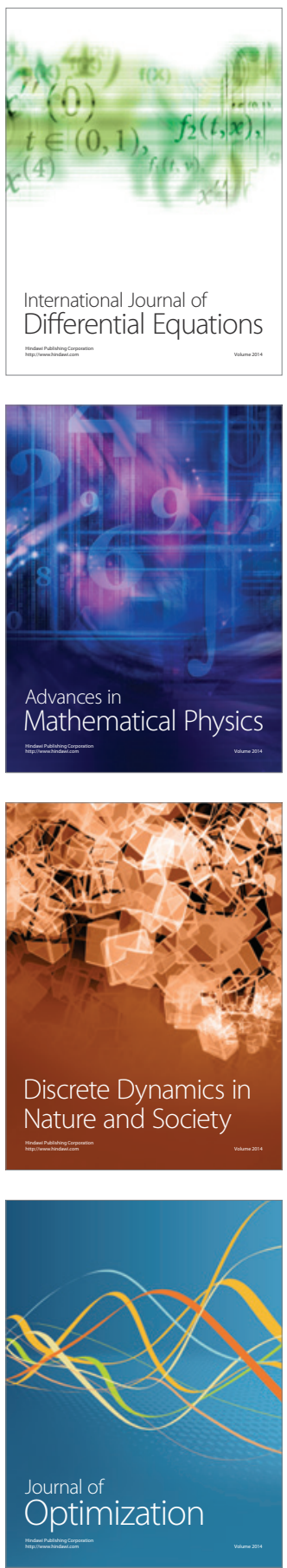